\title{
Mouse Tenm4 is required for mesoderm induction
}

\author{
Hisashi Nakamura, Rita N Cook and Monica J Justice
}

\begin{abstract}
Background: Tenm4 is a mouse homolog of the Drosophila gene Tenascin-m (Ten-m (Odd oz)), which functions in motor neuron routing. Recently, a genome-wide association analysis for bipolar disorder identified a new susceptibility locus at TENM4 increasing the importance of understanding Tenm4. A series of Tenm4 mouse alleles showing a broad range of phenotypes were isolated after ENU mutagenesis. Here, we examine the timing and features of gastrulation failure in a loss of function allele.

Results: Embryonic mesoderm did not form in loss of function Tenm $4^{\mathrm{m} 1 / \mathrm{ml} 1}$ mutant embryos. Genes normally expressed in embryonic mesoderm were not expressed in the mutant, the primitive streak did not form, and markers of the anteroposterior axis were not expressed or were mislocalized. The lack of embryonic mesoderm could not be attributed to poor proliferation of the epiblast, as normal numbers of dividing cells were observed. Epiblast cells maintained expression of Pou5f1 suggesting that they remain pluripotent, but they did not have the capacity to form any germ layer derivatives in teratomas, showing that the inability to induce mesoderm is cell autonomous.

Misexpression of E-cadherin and $\mathrm{N}$-cadherin suggest that the embryos did not undergo an epithelial-to-mesenchymal transition. In addition, Wnt signaling did not occur in the mutants, as assessed by the TOPGAL reporter assay, while a GSK3 $\beta$ inhibitor partially rescued the mutant embryos, and rescued TOPGAL reporter expression.

Conclusions: These data demonstrate that Tenm4 mutants fail to form a primitive streak and to induce embryonic mesoderm. Markers of anterior posterior patterning fail to be expressed or are mislocalized. Further, Tenm4 mutants lack the ability to differentiate in a cell autonomous manner. Together, our data suggest that embryos become impaired prior to E6.5 and as a result, Wnt signaling fails to occur; however, the involvement of other signaling pathways remains to be examined.
\end{abstract}

Keywords: Mouse, Tenm4, Gastrulation, Mesoderm induction, Anterior-posterior patterning, Bipolar disorder, Wnt signaling and ENU mutagenesis

\section{Background}

Tightly controlled signaling pathways play critical roles in the early stages of embryogenesis and body plan formation. During gastrulation, morphogenetic movements in addition to cell proliferation and differentiation transform an embryo with a single germ layer into one with three layers, the ectoderm, mesoderm and endoderm. In the mouse, gastrulation initiates at E6.5, as cells of the primitive ectoderm (epiblast) delaminate at the posterior side of the embryo and ingress through the primitive streak in an epithelial-to-mesenchymal transition [1,2]. Genetic approaches have identified many genes that play a role in gastrulation and/or pattern formation [3]. The evolutionarily conserved signal transduction cascade of $\mathrm{Wnt} / \beta$ -

\footnotetext{
* Correspondence: mjustice@bcm.edu

Department of Molecular and Human Genetics, Baylor College of Medicine, Houston, Texas 77030, USA
}

\section{Biomed Central}

catenin regulates patterning of the visceral endoderm, the induction of the primitive streak, and the formation of anterior neural ectoderm [4,5]. Nodal and Brachyury (T) are among the genes downstream of Wnt signaling that are required for primitive streak formation [5-8].

Tenm4, teneurin transmembrane protein 4 , is a mouse homolog of the Drosophila gene tenascin major (Ten-m), also called $O d d o z(O d z)$ [9-11], which was originally associated with segmentation defects. Ten-m is a member of the Teneurin protein family and encodes a transmembrane protein with tenascin-like EGF (epidermal growth factor) repeats. Ten- $m$ is found in both secreted and membrane-bound forms at several compartment boundaries, suggesting a role in cell-cell communication. $\mathrm{Mu}-$ tant Ten-m Drosophila embryos have abnormalities in neural ectoderm and mesoderm-derived tissues that result in death at the larval stage $[9,10]$. Recently, new 
alleles of Drosophila Ten- $m$ were identified and characterized establishing that mutations in this gene do not cause segmentation defects; instead, Ten- $m$ functions in motor neuron routing [12]. Ten-m is expressed in the central nervous system and epidermal stripes at stages when the growth cones of intersegmental neurons (ISNs) navigate to their targets. Both mutation and over-expression of Ten-m in epidermal cells leads to ISN misrouting [12]. A related protein, tenascin a (Ten-a) has a trans-synaptic signaling role with Ten-m: Ten-a is presynaptic whereas Ten$\mathrm{m}$ is predominantly postsynaptic in neuromuscular synapse organization and target selection [13].

The mouse has four teneurin transmembrane protein family members (Tenm1-4) that lack signal peptides at the $\mathrm{N}$-terminus, but contain a short hydrophobic domain characteristic of transmembrane proteins followed by a region with eight EGF-like repeats, and a large C-terminal domain $[14,15]$. The vertebrate homolog of Drosophila Ten- $a$ is called Tenm1 and the homolog of Drosophila Ten-m is Tenm4. All four mammalian Tenm genes are highly expressed in the brain and each gene produces many alternatively spliced transcripts, suggesting a variety of protein functions in different tissues [16,17]. Novel mutant alleles having defects in early mouse embryonic development can be identified using ethylnitrosourea (ENU) mutagenesis [18]. A series of ENU-induced alleles at mouse 17Rn3 [19-21] contain mutations in the Tenm4 gene [16], which exhibit a wide array of phenotypes, ranging from embryonic death at gastrulation to viable with skeletal defects [16]. In two loss-of-function alleles $\left(T e n m 4^{m 1}\right.$ and Tenm $4^{m 2}$ ), embryos fail to gastrulate. In less severe alleles $\left(\right.$ Tenm $4^{m 3}{ }^{\text {, Tenm }} 4^{m 4}$ and Tenm $4^{m 5}$ ), gastrulation occurs, but body axis formation, somitogenesis, vasculogenesis, cardiogenesis, and fusion of the allantois with the chorion are disrupted leading to death at earlyto mid-gestation stages. Consistent with these mutant phenotypes, Tenm4 is ubiquitously expressed in the epiblast and extraembryonic regions as early as E6.5. By E7.5, Tenm4 is highly expressed in the mesoderm of the developing embryo and extraembryonic tissues. Later, Tenm4 is expressed mainly in the neuroectoderm, but expression is maintained in the tail bud, somites and limbs [16].

To begin to address the biological function of Tenm4, the loss of function allele Tenm $4^{m 1}$, and a hypomorphic allele Tenm $4^{m 4}$ were examined. Tenm $4^{m 1 / m 1}$ mutant embryos failed to initiate gastrulation, failed to form a primitive streak, and failed to develop mesoderm. In addition, Tenm $4^{m 1 / m 1}$ mutant embryos were incapable of forming any differentiated tissue. An analysis of the hypomorphic allele, Tenm $4^{m 4}$, along with the null allele, determined that mutant cells did not properly express Eor N-cadherin, suggesting that the epithelial-to-mesenchymal transition (EMT) did not occur. Moreover, the mutants failed to up-regulate a TOPGAL reporter gene, suggesting that Wnt signaling failed to occur. Finally, a GSK3 $\beta$ inhibitor rescued the ability of the embryos to form extraembryonic mesoderm and partially rescued their ability to form embryonic mesoderm.

\section{Results}

Tenm $4^{\mathrm{m} 1 / \mathrm{m} 1}$ mutant embryos arrest at the gastrulation stage

At E6.5, wildtype and Tenm $4^{m 1 / m 1}$ embryos were similar in appearance (Figure 1A and D). At E7.5, mutant

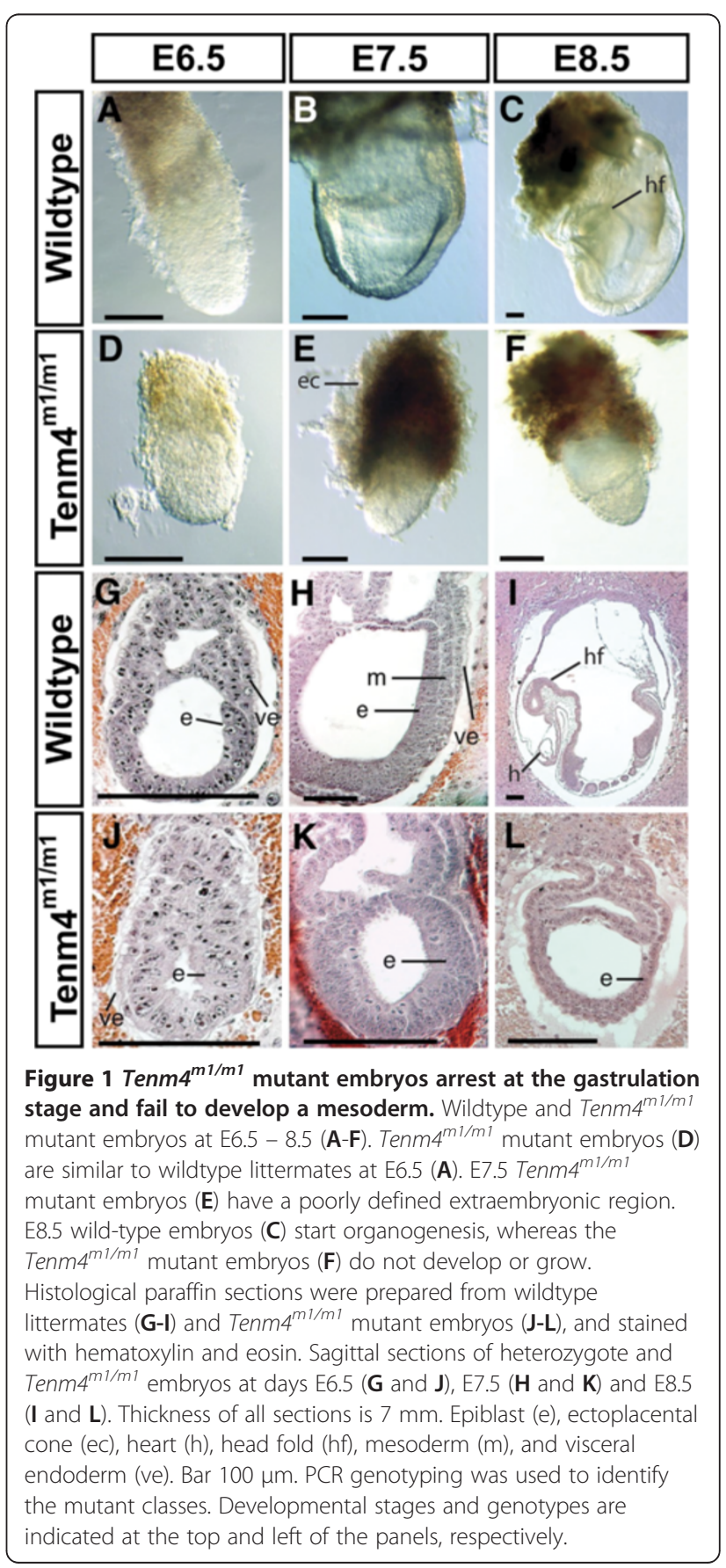


embryos were approximately half the size of littermates (Figure $1 \mathrm{~B}$ and $\mathrm{E}$ ), and at E8.5, mutant embryos were arrested in development at the E6.5 stage (Figure $1 \mathrm{C}$ and F). Prior studies showed that most embryos were dead or dying by E8.5 and that no mutant embryos survived past E9.5 [16]. Histology revealed phenotypic differences between wildtype and mutant embryos (Figure 1G-L). At E6.5 wild-type embryos had a well-organized ectoderm, visceral endoderm, and extraembryonic mesoderm with proamniotic and extraembryonic cavities (Figure 1G). In contrast, mutant embryos had no sign of mesoderm (Figure 1J). At E7.5, wildtype embryos developed embryonic mesoderm (Figure 1H). By E8.5, wildtype embryos developed three primitive embryonic cavities, including the amniotic, exocoelomic and ectoplacental cavities, head folds, and embryonic and extraembryonic mesodermal tissues, including the allantois (Figure 1I). However, no mesoderm formed and the embryonic region did not expand in Tenm $4^{m 1 / m 1}$ mutant embryos by E7.5 or 8.5 , although some embryos appeared to form a morphologically abnormal extraembryonic cavity, perhaps caused by the expansion of the extraembryonic ectoderm and visceral endoderm (Figure $1 \mathrm{~K}$ and $\mathrm{L}$ ).

\section{Tenm $4^{\mathrm{m} 1 / \mathrm{m} 1}$ mutants do not produce mesoderm}

Brachyury is expressed prior to the onset of gastrulation at E6.5 in the extraembryonic ectoderm adjacent to the epiblast, and shifts to the developing primitive streak, where it is a marker of mesodermal-derived notochord [22,23]. No Brachyury expression was found in the embryo of Tenm $4^{m 1 / m 1}$ mutants, although a weak signal was observed in the extraembryonic tissue in half of the mutants (Figure 2A, F and Additional file 1: Figure S1). The cause of the inconsistent extraembryonic expression is not clear. This is an ENU-induced point mutation, and often such alleles are somewhat leaky due to read-through of the point mutation. Alternatively, this observation is consistent with expression in extraembryonic ectoderm, indicating developmental delay. Importantly, none of the mutants had Brachyury expression in the area of the potential primitive streak. Additional primitive streak markers were examined. In wildtype embryos, Foxa2 $(H n f 3 \beta)$ expression is restricted to the anterior end of the primitive streak at E7.0 (Figure 2B and [24-26]. $L h x 1$ is expressed at low levels in cells entering the streak and at high levels in cells exiting the streak, and similarly, in anterior endoderm at E7.5 (Figure 2C and [26,27]. In Tenm $4^{m 1 / m 1}$ mutants, neither Foxa 2 nor $\operatorname{Lh} x 1$ expression was detected (Figure $2 \mathrm{G}$ and $\mathrm{H}$, respectively).

To determine how the Tenm $4^{m 1}$ mutation affects the development of the anterior patterning of the embryo, the expression pattern of Otx2 was examined, which is expressed uniformly in the epiblast and the anterior visceral endoderm (AVE) prior to gastrulation. The expression of Otx2 becomes restricted to the anterior epiblast as mesoderm migrates from the primitive streak suppressing its expression in the posterior epiblast (Figure 2D and [28]). In Tenm $4^{m 1 / m 1}$ embryos, Otx2 expression does not become fully restricted to the anterior epiblast, consistent with the lack of mesoderm influencing its

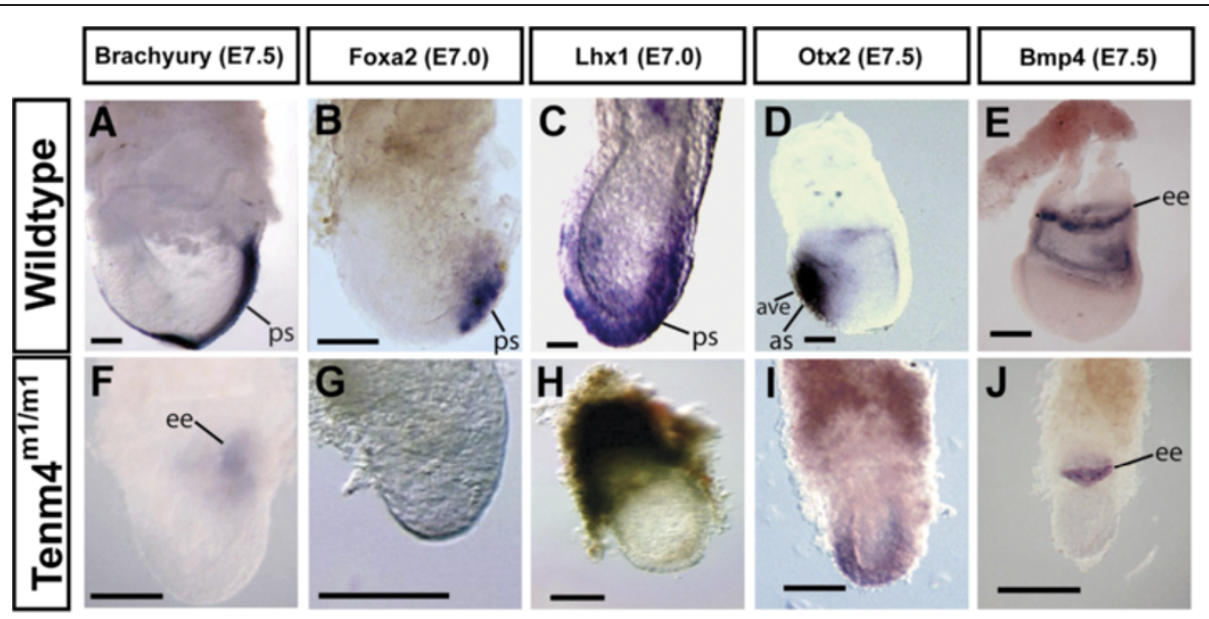

Figure 2 Mesodermal and anterior marker gene expression in Tenm $4^{\mathbf{m} 1 / m} \mathbf{m}$ mutant embryos. Whole-mount in situ hybridization was performed to examine the expression of marker genes (Brachyury, Foxa2, Lhx1, Otx2 and Bmp4). Wildtype embryos are oriented with the anterior side of embryos on the left. A-P axis orientation may vary because the A-P axis was not specified in mutants. Brachyury is expressed in the primitive streak and notochord of an E7.5 wildtype embryo (A). Tenm $4^{\mathrm{m} 1 / \mathrm{m} 1}$ mutants at E7.5 lack Brachyury expression in the posterior embryonic region (F), although a weak signal is detected in extraembryonic tissue. Foxa2 is expressed in the primitive streak in an E7.0 wildtype embryo (B), but not in Tenm $4^{m 1 / m 1}(\mathbf{G})$. Lhx1 is expressed in mesoderm wings and anterior visceral endoderm at E7.0 (C), but not in Tenm4 ${ }^{\mathrm{m} 1 / \mathrm{ml}}$ ( $\left.\mathbf{H}\right)$. Otx2 is restricted to the anterior epiblast of an E7.5 wildtype embryo (D), but not restricted in Tenm4 ${ }^{\mathrm{m} 1 / \mathrm{ml}}$ (I). Bmp4 is expressed in extraembryonic ectoderm and extraembryonic mesoderm at E7.5 (E). Tenm4 ${ }^{\mathrm{m} 1 / \mathrm{m} 1}$ mutant shows ectodermal expression but not mesodermal (J). Anterior streak (as), anterior visceral endoderm (ave), extraembryonic ectoderm (ee), primitive streak (ps). Bar $100 \mu \mathrm{m}$. 
restricted expression (Figure 2I). Bmp4 is expressed in the extraembryonic ectoderm adjacent to the proximal epiblast prior to gastrulation and later in the posterior primitive streak and extraembryonic mesoderm (Figure 2E and [29]. In Tenm $4^{m 1 / m 1}$ mutants, the expression of Bmp4 is limited to a proximal ring at the junction of the embryonic and extraembryonic ectoderm, confirming the absence of posterior mesoderm (Figure 2J).

\section{Proliferation and apoptosis of epiblast cells in Tenm $4^{\mathrm{m} 1 / \mathrm{m} 1}$ is similar to wild type}

A threshold number of epiblast cells must be attained and maintained for gastrulation to initiate and progress [3]. Mouse embryos that lack a normal number of cells due to abnormal cell proliferation or to cell losses will delay gastrulation until the embryo attains the appropriate number of cells. Mutant embryos that fail to sustain the proliferation of epiblast cells do not initiate gastrulation or will arrest after the formation of a rudimentary primitive streak. To examine the effect of the Tenm $4^{m \boldsymbol{1}}$ mutation on cellular proliferation in vivo, the incorporation of $5^{\prime}$-bromo- $2^{\prime}$ - deoxyuridine (BrdU) into DNA was analyzed. At E6.5 (Figure 3A, B and Table 1), 48\% of the nuclei of wildtype or heterozygous epiblast cells were labeled with BrdU after a 20-minute exposure. In Tenm $4^{m 1 / m 1}$ mutant embryos, $47 \%$ of the nuclei were labeled, suggesting that the proliferation of epiblast cells is normal at this time point. These data suggest that the gastrulation defect is not caused by insufficient cell proliferation in the epiblast. However, the total number of mutant epiblast cells was lower at E6.5 (Figure 3B and Table 1), and a student's t-test indicates that total cell numbers are significantly different $(t=0.002)$. This suggests that the growth of the embryo starts to become impaired prior to E6.5. Increased apoptosis could also cause a reduced number of cells, so the TUNEL assay was used to examine cell death. Normally, cell death occurs primarily in extraembryonic tissues, especially in trophoblast (Figure $3 C)$, but is rare in embryonic cells. Very few apoptotic cells were observed in mutant embryos at E6.5 (Data not shown) or at E7.5 (Figure 3D and Table 1).

The epiblast contains a highly regulated stem cell population that adjusts to various perturbations including major

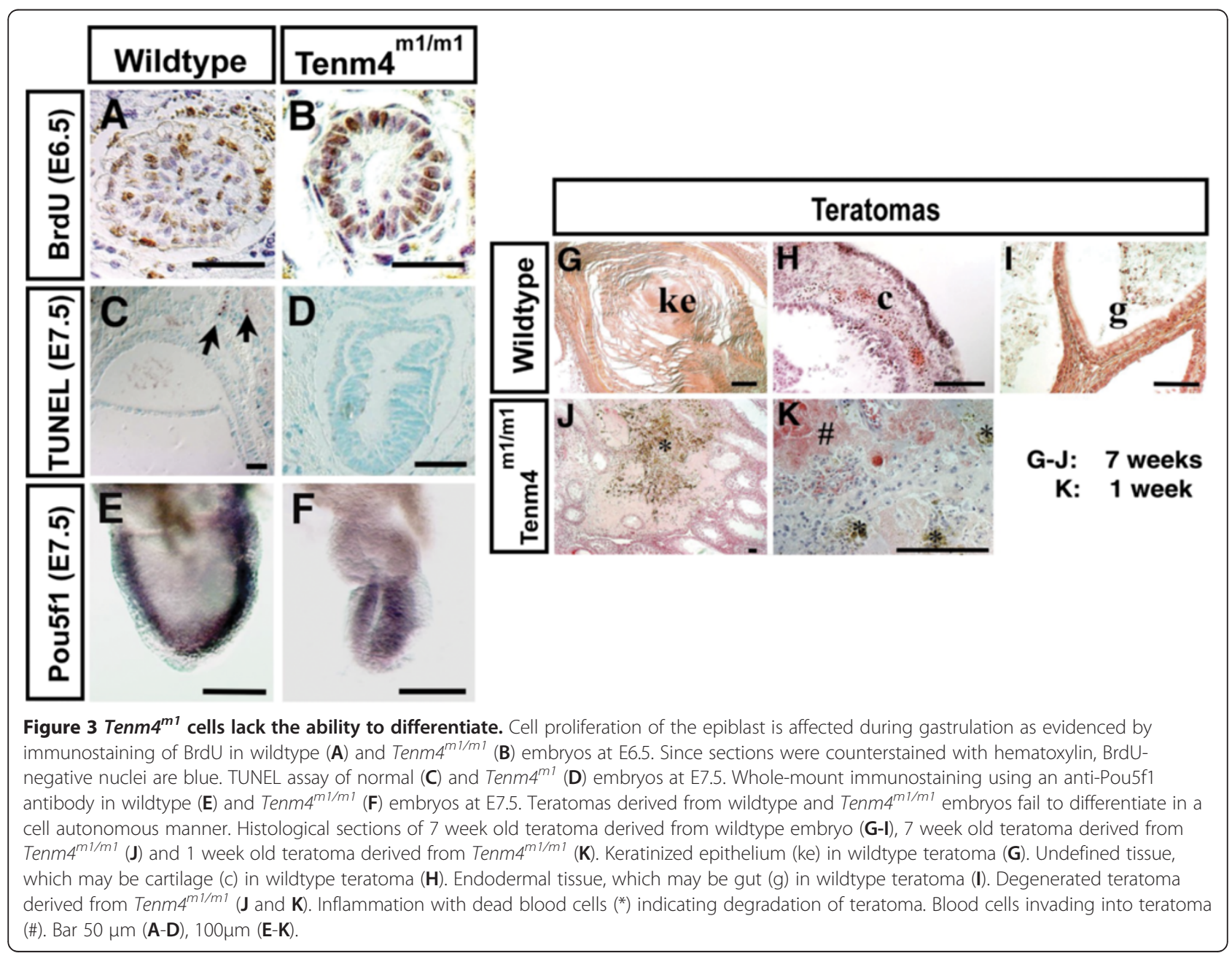


Table 1 Tenm $4^{m 1 / m 1}$ mutant embryo BrdU incorporation and TUNEL assay results

\begin{tabular}{|c|c|c|}
\hline i) BrdU (E6.5) & wt or $m 1 /+(n=24)$ & $m 1 / m 1(n=13)$ \\
\hline Total cells (per section) & $88.2( \pm 11.5)$ & $62.2( \pm 4.66)$ \\
\hline BrdU labeled cells & $42.1( \pm 6.41)$ & $29.2( \pm 3.67)$ \\
\hline Labeling index (\%) & $47.7( \pm 4.45)$ & $46.9( \pm 2.59)$ \\
\hline ii) TUNEL assay (E7.5) & wt or $m 1 /+(n=10)$ & $\mathrm{m} 1 / \mathrm{m} 1(\mathrm{n}=6)$ \\
\hline Embryonic region & $1.2( \pm 0.44)$ & $0.17( \pm 0.41)$ \\
\hline Extraembryonic region & $11.3( \pm 3.11)$ & $0.50( \pm 0.84)$ \\
\hline
\end{tabular}

i) BrdU incorporation at E6.5. ii) TUNEL assay at E7.5. Numbers in parentheses are standard deviation $( \pm \mathrm{SD})$

alterations in cell number and cell position. Because the cells remain in an undifferentiated state until late gastrulation $[30,31]$, one possibility for the low cell count in Tenm $4^{m 1 / m 1}$ at the late gastrulation stage is a precocious differentiation of epiblast stem cells. Therefore, the expression of Pousf1 at E7.5 was examined. Pousf1 is distinguished by exclusive expression in blastomeres, pluripotent cells, and the germ cell lineage [32-36]. At E7.5, Pou5f1 expression should be restricted to the epiblast (Figure 3E). In mutant embryos, the expression of Pousf1 is similar to wildtype, indicating that undifferentiated pluripotent epiblast cells are present (Figure 3F).

To examine differentiation potential, wildtype and mutant embryonic tissues at E6.5 were transplanted into the testes of adult male mice and examined for the development of teratomas. After 7 weeks, the testes were fixed, sectioned and processed for histological analysis (Figure 3 G-K). Teratomas derived from heterozygotes differentiated into many kinds of tissues, which are produced from all three germ layers (Figure 3G-I). However, the teratomas derived from Tenm $4^{m 1 / m 1}$ mutant embryos produced no differentiated tissues (Figure 3J). The size of teratomas derived from mutant embryos never exceeded $2 \mathrm{~mm}$, whereas teratomas from wildtype embryos grew to $0.5-2$ $\mathrm{cm}$ in diameter (Table 2). Furthermore, approximately half of the mutant-derived teratoma cells had degenerated when examined at 7 weeks. Only four homozygous mutant-derived teratomas survived to be genotyped out of 43 teratomas examined (9.3\%), all of which had inflammatory reactions, indicating the decay of the cells. Moreover, six teratomas could not be genotyped because they were severely decomposed. Mutant-derived teratomas had signs of inflammation when teratomas were assessed at 1 week after transplantation, although again, no cell differentiation was observed (Figure 3K). Together, these results suggest that the failure to differentiate is cell autonomous. To further test this question, embryos were cultured to attempt to generate embryonic stem (ES) cells for studies of chimeras with mutant cells. Although multiple ES cell lines were derived from wildtype $(4 / 55)$ or heterozygous $(12 / 55)$ embryos, none were derived from mutants $(0 / 55)$,
Table 2 Teratomas ( 7 weeks old and 1 week old) derived from Tenm $4^{m 1}$ mutant embryos

\begin{tabular}{|c|c|c|c|}
\hline \multicolumn{4}{|l|}{ i) 7 weeks old } \\
\hline & $+/+$ & $\mathrm{m} 1 /+$ & $\mathrm{m} 1 / \mathrm{m} 1$ \\
\hline No. of transplantation & 9 & 24 & 10 \\
\hline Tumor developed & 9 & 24 & 4 \\
\hline Dead or degrading & 0 & 0 & $6^{*}$ \\
\hline \multicolumn{4}{|l|}{$\underline{\text { Tumor size }}$} \\
\hline large $(1.0-2.0 \mathrm{~cm})$ & 8 & 24 & 0 \\
\hline middle $(0.5-1.0 \mathrm{~cm})$ & 1 & 0 & 0 \\
\hline small $(<0.5 \mathrm{~cm})$ & 0 & 0 & 4 \\
\hline \multicolumn{4}{|l|}{$\underline{\text { Differentiation }}$} \\
\hline Ectoderm & 9 & 24 & 4 \\
\hline Mesoderm & 8 & 21 & 0 \\
\hline Endoderm & 4 & 14 & 2 \\
\hline \multicolumn{4}{|l|}{ ii) 1 week old } \\
\hline & $+/+$ & $\mathrm{m} 1 /+$ & $\mathrm{m} 1 / \mathrm{m} 1$ \\
\hline No. of transplantation & 3 & 8 & 5 \\
\hline Tumor developed & 3 & 7 & 5 \\
\hline Dead or degrading & 0 & 1 & 0 \\
\hline \multicolumn{4}{|l|}{$\underline{\text { Tumor size }}$} \\
\hline large $(1.0-2.0 \mathrm{~cm})$ & 0 & 0 & 0 \\
\hline middle $(0.5-1.0 \mathrm{~cm})$ & 2 & 5 & 0 \\
\hline small $(<0.5 \mathrm{~cm})$ & 1 & 2 & 5 \\
\hline \multicolumn{4}{|l|}{$\underline{\text { Differentiation }}$} \\
\hline Ectoderm & 3 & 7 & 0 \\
\hline Mesoderm & 2 & 5 & 0 \\
\hline Endoderm & 2 & 4 & 0 \\
\hline
\end{tabular}

Teratomas were assessed by histology after measuring the diameter of the teratoma. The teratoma sizes were categorized as large $(1-2 \mathrm{~cm})$, middle $(0.5-1$ $\mathrm{cm}$ ) and small (less than $0.5 \mathrm{~cm}$ ) and the tissue types in teratomas derived from normal and mutant embryos were categorized as ectoderm, endoderm and mesoderm. ${ }^{*}$ Not genotyped due to degrading of teratoma tissue.

precluding experiments on such cells. Blastocysts from matings of Ten- $m 4^{m 1}$ heterozygotes were collected at E3.5 and cultured in vitro to observe any defects in the inner cell mass (ICM) and/or trophectoderm outgrowth. All blastocysts attached normally to gelatin-coated dishes and completely hatched from the zona pellucida after $2-3$ days in culture. After hatching, the growth of the ICM and the extent of trophoblast outgrowth was very abnormal in Ten-m $4^{m 1 / m 1}$ mutants (Additional file 2: Figure S2). Mutant cells could be easily distinguished from those from wild type or heterozygotes because they failed to adhere and could not survive more than 8 days in culture.

\section{Expression of E-cadherin, N-cadherin and Snai1}

An epithelial-to-mesenchymal transition (EMT) is an essential step in gastrulation, which requires Wnt signaling to proceed. Down-regulation of E-cadherin is an early 
step of the EMT. Embryos that fail to gastrulate do not undergo the EMT; however, the Tenm4 allelic series includes a hypomorphic allele, Tenm $4^{m 4 / m 4}$, which gastrulates to form mesodermal tissues, including yolk sac, heart and blood vessels, yet arrests in development by E8.5 [16]. The m4 allele allows the EMT to be assessed in a mutation that does not arrest before gastrulation occurs. The expression of E-cadherin in the loss of function allele Tenm $4^{m 1 / m 1}$ and the hypomorphic allele Tenm $4^{m 4 / m 4}$ was examined. E-cadherin was absent in the primitive streak and mesoderm of wildtype embryos, (Figure 4B), whereas E-cadherin expression was not down regulated in the epiblast of both Tenm $4^{m 1 / m 1}$ and Tenm $4^{m 4 / m 4}$ (Figure $4 \mathrm{C}$ and D, respectively). When epiblast cells delaminate at the primitive streak to form mesoderm, they switch expression from $\mathrm{E}$ - to $\mathrm{N}$ - cadherin. $\mathrm{N}$-cadherin was expressed at high levels in the yolk sac and primitive streak in wildtype embryos (Figure 4E); however, no $\mathrm{N}$-cadherin expression was observed in either
Tenm $4^{m 1 / m 1}$ or Tenm $4^{m 4 / m 4}$ (Figure $4 \mathrm{~F}$ and G). Snai1 (mSnail) suppresses E-cadherin to initiate migration of trophoblast giant cells, which also requires the EMT. No Snail expression was observed in cultured ectoplacental cone mutant explants (Figure $4 \mathrm{H}$ and I). All wildtype explants expressed Snai1 (100\%; n=15), whereas only one Tenm $4^{m 1 / \mathrm{m} 1}$ explant had low Snail expression, and five had none $(\mathrm{n}=6), 16.7 \%$.

\section{The TOPGAL assay suggests that Wnt signaling does not occur}

Drosophila Ten-m affects wingless expression [9]. In mouse, canonical signaling by Wnt3 is required for mesoderm induction in the early embryo [5]. A TOPGAL transgenic expression reporter mouse strain, which expresses $\beta$-galactosidase under the control of three copies of the Wnt-specific LEF/TCF binding sites was used to assess Wnt signaling in the mutants [37]. In wild-type E7.5 embryos, TOPGAL activity was detected
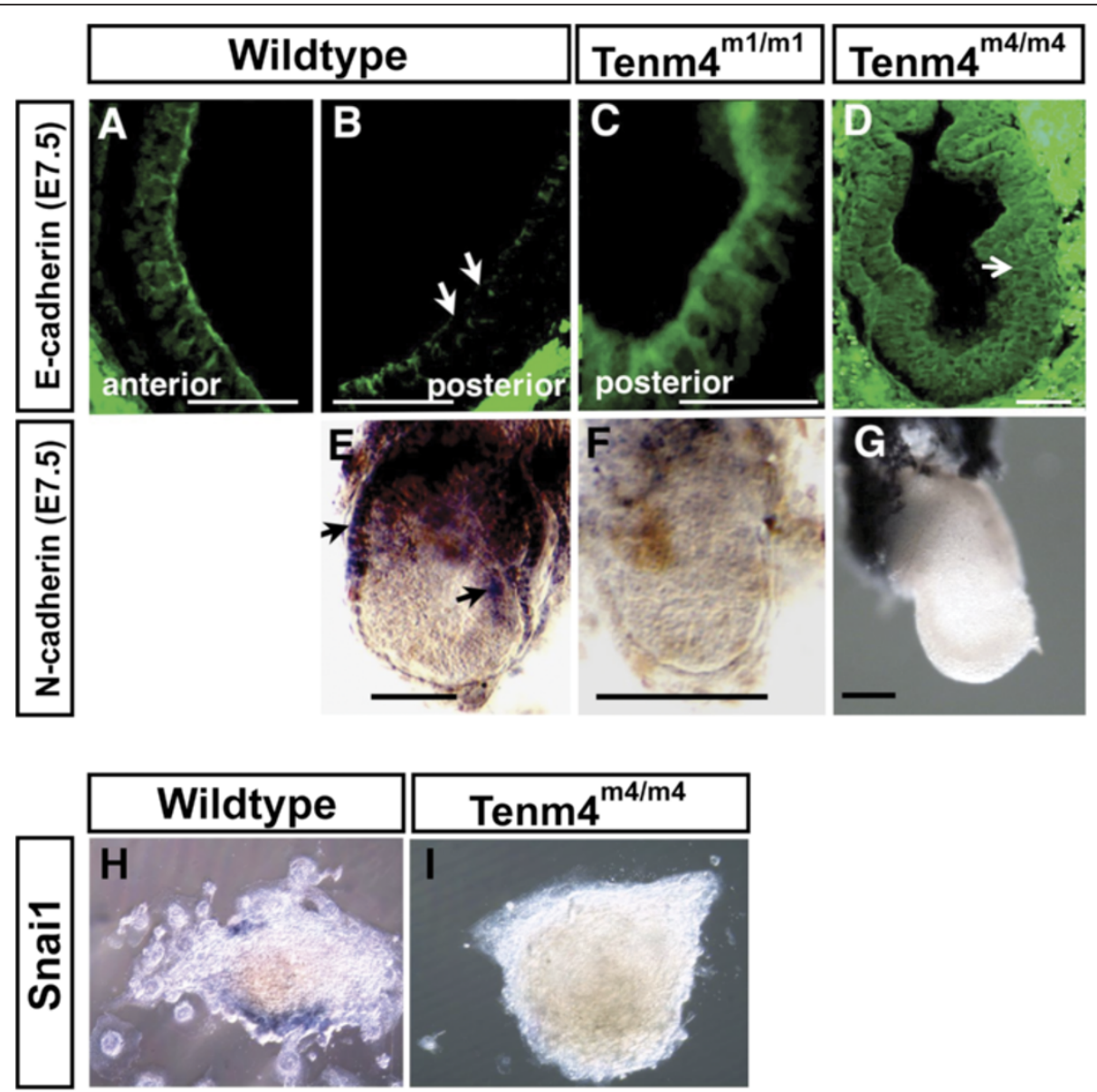

Figure 4 E-cadherin, $\mathbf{N}$-cadherin and Snai1 expression. E-cadherin is down-regulated in the primitive streak and mesoderm in wildtype embryos (B, white arrows), but not in Tenm $4^{m 1 / m 1}$ (C) or Tenm $4^{m 4 / m 4}$ (D). Note the formation of mesoderm in the m4 allele (white arrow), while E-cadherin fails to be down-regulated. N-cadherin is expressed in the visceral endoderm and the primitive streak in wildtype embryos (E); however, neither Tenm $4^{\mathrm{ml} / \mathrm{ml}}$ nor Tenm $4^{\mathrm{m} 4 / \mathrm{m} 4}$ mutants show expression of N-cadherin (F and $\mathbf{G}$ ). Snai1 is expressed in ectoplacental cone

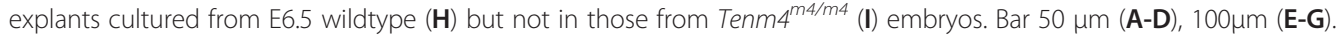


in the primitive streak (Figure $5 \mathrm{~A}$ and $\mathrm{B}$ ). In contrast, TOPGAL activity was not present in Tenm $4^{m 1 / m 1}$ E7.5 embryos (Figure 5D).

Normally, glycogen synthase kinase 3 (GSK3) is inhibited by Wnt signaling to promote stabilization of $\beta$-catenin and transcription of target genes [38]. To attempt to rescue the defective mesoderm induction in Tenm $4^{m 1 / m 1}$ mutant

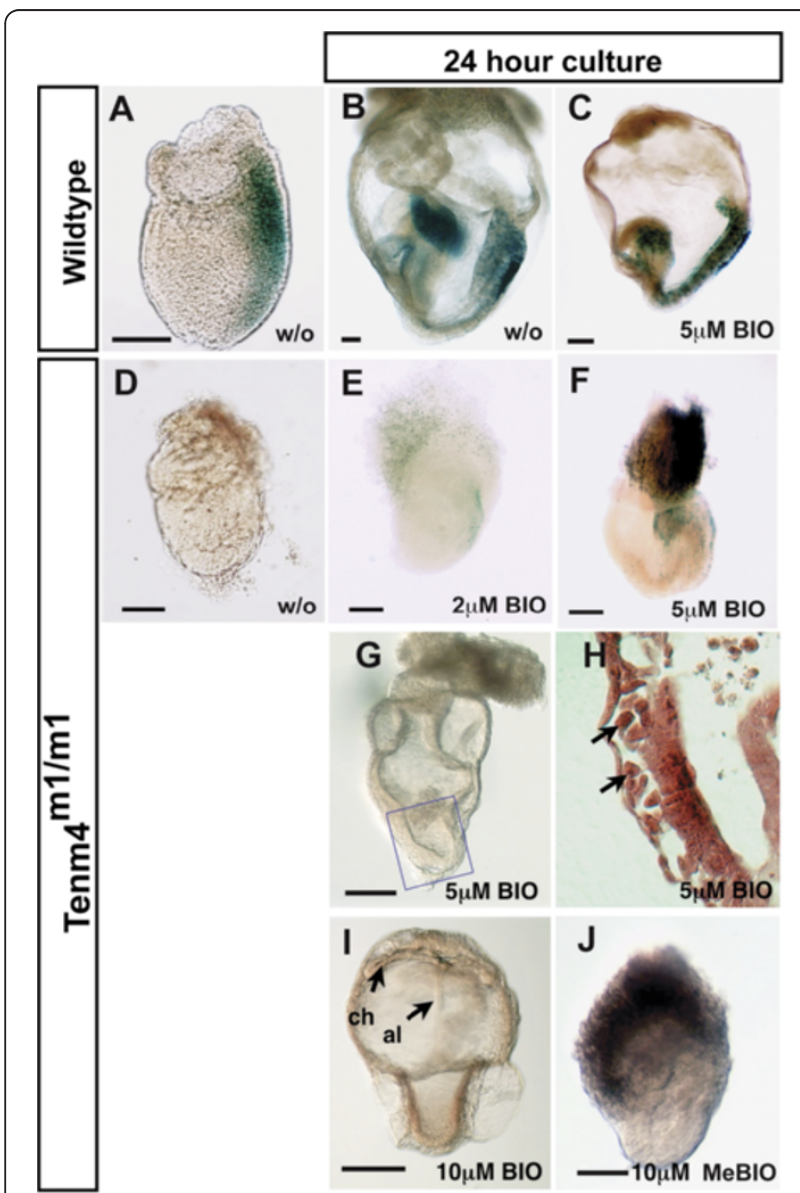

Figure 5 Partial rescue of TOPGAL reporter expression by GSK inhibitors in Tenm $4^{\mathrm{m} 1 / \mathrm{m} \mathbf{1}}$ mutants. Shown are E7.5 embryos removed from the maternal environment, then cultured for 24 hours without addition of $\mathrm{BIO}$ or $\mathrm{MeBIO}$ (w/O), with 2, 5 or $10 \mu \mathrm{M} 6$ bromoindirubin-3'-oxime (BIO), or 10 MM 1-methyl-6-bromoindirubin-3'oxime (MeBIO). The TOPGAL reporter transgene was used to assess WNT signaling by staining for $\beta$-galactosidase activity. Wildtype embryos obtained at E7.5 (A) and cultured for 24 hours (B and $\mathbf{C}$ ) had $\beta$ galactosidase expression without or with the addition of BIO. Tenm $4^{\mathrm{ml} / \mathrm{m} 1}$ mutant embryos had no $\beta$-galactosidase expression when obtained at E7.5 (D) but expressed $\beta$-galactosidase after 24 hours in $2 \mu \mathrm{M}(\mathbf{E})$ or $5 \mu \mathrm{M}$ $\mathrm{BIO}(\mathbf{F})$. A Tenm $4^{\mathrm{m} 1 / \mathrm{ml}}$ mutant embryo cultured 24 hours in $5 \mu \mathrm{M} \mathrm{BIO}$, not stained for $\beta$-galactosidase to show morphological features is shown in $\mathbf{G}$ alongside a histological section $(\mathbf{H})$ from the area in the blue box showing migrating mesodermal cells (arrowheads). A Tenm $4^{\mathrm{ml} / \mathrm{ml}}$ mutant embryo cultured 24 hours with $10 \mu \mathrm{M}$ BIO shows fusion of the chorion (ch) with the allantois (al) (I), also not stained with $\beta$ galactosidase. No embryos cultured with $\mathrm{MeBIO}$ showed evidence of mesoderm or TOPGAL activity, a representative one cultured in 10uM MeBIO is shown in (J). Bar $100 \mu \mathrm{m}$. embryos, Wnt signaling was ectopically induced with a GSK3 specific inhibitor, 6-bromoindirubin-3'-oxime (BIO). Its analog, 1-methyl-6-bromoindirubin-3'-oxime (MeBIO) is a useful negative control [39]. When MeBIO was added to the embryo culture medium, Tenm $4^{m 1 / m 1}$ mutant embryos did not produce any mesodermal cells (Figure 5J). However, when BIO was added at $2 \mu \mathrm{M}$ and $5 \mu \mathrm{M}$, extraembryonic tissue grew (Figure 5E, F and G). Even so, the embryonic region did not expand, although some cells migrated away from the epiblast (Figure $5 \mathrm{G}$ and $\mathrm{H}$ ). Using a higher BIO concentration $(10 \mu \mathrm{M})$, Tenm $4^{m 1 / m 1}$ mutant embryos produced allantois and chorion, which fused, suggesting that extraembryonic mesoderm was produced and differentiated (Figure 5I). Moreover, TOPGAL signaling was induced to various degrees in both embryonic and extraembryonic tissues (Figure 5E, F, G and I), suggesting that Wnt signaling was restored in some cells.

\section{Discussion}

Here we show using mouse mutants that the earliest functions of Tenm4 are prior to gastrulation such that Wnt signaling does not occur. A loss of function allele failed to gastrulate and produced no mesoderm. In vivo and in vitro experiments showed that loss of function mutant embryos did not have the potential to form differentiated tissues, a defect that was cell autonomous. Further, E-cadherin and N-cadherin expression was abnormal in both loss of function and hypomorphic alleles, supporting the idea that Tenm4 mutant cells fail to undergo the epithelial-to-mesenchymal transition, surprisingly even when a primitive streak forms and gastrulation occurs. The formation of embryonic cavities, along with weak expression of Brachyury in extraembryonic regions and rescue of extraembryonic mesoderm by GSK inhibitors, suggests that extraembryonic mesoderm may remain competent in the mutants; however, mesoderm in the embryo proper may lack the potential to differentiate.

The phenotype of Tenm $4^{m 1 / m 1}$ mutant embryos is distinct from other mice with gastrulation failure. Mutations in Nodal, Smad2, Smad4 or Actr1b, which block signaling of members of the transforming growth factor beta (TGF- $\beta$ ) family, affect embryonic differentiation at the egg cylinder stage before gastrulation [6,40-43]. Smad2-dependent Nodal/activin/TGF- $\beta$ signaling is essential for the maintenance of pluripotency in the epiblast and in human embryonic stem cells [44]. Tenm $4^{m 1}$ deficient embryos maintain expression of Pou $5 f 1$ in the epiblast, suggesting that the epiblast has pluripotent potential. Foxh1 and Cripto mediate the Nodal-signaling pathway $[45,46]$. In contrast to Tenm $4^{m 1}$, Foxh1- and Cripto-deficient embryos produce a primitive streak. Bone morphogenic protein (BMP) signaling is also required for gastrulation, and mesoderm induction fails in 
type-I BMP receptor (Bmpr1) mutants [47]. In contrast to Tenm4 mutants, teratomas derived from $\mathrm{Bmpr}^{-1-}$ embryos produce mesoderm-derived tissues [47]. BMP signaling is required for visceral endoderm differentiation and the formation of cavities in the early mouse embryo [48]. Tenm $4^{m 1 / m 1}$ embryos developed normal visceral endoderm and embryonic cavities, as they maintained Bmp4 expression in the extraembryonic ectoderm. Taken together, these data suggest that the first embryonic function of Tenm4 may not be to target TGF- $\beta$ or BMP signaling.

Canonical Wnt signaling is essential for mesoderm formation, embryonic patterning, and epithelial-to-mesenchymal interactions. Wnt3 plays a role in inducing mesoderm and forming the primitive streak $[49,50]$. Although Wnt3 mutants fail to induce mesoderm, the visceral endoderm and epiblast layers continue to grow and expand [5]. In contrast, Tenm $4^{m 1 / m 1}$ embryos failed to expand the visceral endoderm and epiblast layers. Mesd, which encodes the chaperone for the Wnt co-receptors LRP5/6, as well as a double knockout of LRP5/6, shows phenotypes similar to the Tenm $4^{m 1}$ mutant $[49,50]$. In these mutants, defects in extraembryonic tissues lead to failure to organize the proximal epiblast, similar to Tenm4; however, in contrast, the visceral endoderm and epiblast layers continue to grow, similar to Wnt3 mutants. $\beta$-catenin regulates Cripto- and Wnt3-dependent gene expression programs in mouse anterior posterior axis and mesoderm formation, controlling both the Wnt and nodal pathways [51-53]. No mesoderm or head structures formed in $\beta$-catenin-deficient embryos, and markers of posterior mesoderm differentiation such as Brachyury, as well as markers of A-P axis formation such as Hex and Hesx1, were not expressed [54]. Similar to $\beta$-catenin- and Wnt3-deficient embryos, Tenm $4^{m 1 / m 1}$ mutants failed to form a primitive streak, even though the epiblast maintained high Pou5f1 expression, unlike mutations in Smad2, Smad4 and Nodal. The Tenm4 $4^{m 1 / m 1}$ mutant phenotype is more similar to canonical Wnt mutants than those of other pathways, yet seems to lack competency for the embryonic epiblast to differentiate into mesoderm [55].

Fgfr1 mutants that do not activate Snail show defective mesoderm migration and differentiation [56]. The suppressor of E-cadherin, Snai1, was not upregulated in cultured mutant ectoplacental cone explants of the hypomorphic mutant Tenm $4^{m 4}$. These data, combined with knowledge of Tenm family EGF structural motifs, membrane orientation, and known secreted and membrane-bound forms, suggest that Tenm 4 may be an important molecule for proper adhesion of migrating extraembryonic cells. Embryo cultures indicated that a GSK3 $\beta$ inhibitor induced the outgrowth of extraembryonic tissues, and partially rescued defective mesoderm induction, particularly in extraembryonic tissues. The activity of GSK inhibitors is not restricted to Wnt signaling, and in fact, can influence cell adhesion molecules [57]. Furthermore, the inhibitors did not fully rescue the mutant phenotypes, suggesting that other pathways are likely to be involved.

The Wnt pathway has evolved to interact with many receptors in a variety of signaling pathways that are independent of canonical signaling, see [58] for review. For example, non-canonical Wnt signaling is required for planar cell polarity and convergent extension [58]. In addition to its essential roles in gastrulation, stem cell homeostasis and axis patterning, the Wnt pathway plays a key role in neuronal development and maintenance. In particular, non-canonical Wnt signaling is crucial for path finding during axonal navigation as well as for synapse formation [58,59]. Drosophila Ten-m binds with filamins via its filamin-binding domain to reorganize the cytoskeleton during axon guidance [12], a function of non-canonical Wnt signaling. Ten-m is expressed in muscle, but interacts with Ten-a at the neuromuscular synapse, evidencing its role in cell-cell communication during synapse organization and function [13]. Recently, a large-scale genome-wide association study (GWAS) identified an intronic variant in TENM4 (ODZ4) as being associated with bipolar disorder, a severe mood disorder that affects over $1 \%$ of the population [60]. Wnt signaling is implicated in many neuropsychiatric disorders, including schizophrenia, bipolar disorder and autism [59]. Therefore, it will be intriguing to determine if Tenm4 has a direct role in Wnt signaling; however, evidence for this will require the analysis of other Tenm4 alleles, which have abnormal body axis patterning or abnormal neural development [16].

\section{Conclusions}

Our data show that Tenm4 is required for mesoderm induction during gastrulation, and that gastrulation arrests at a stage prior to that in which Wnt signaling occurs. Tenm $4^{m 1 / m 1}$ mutants have a phenotype that is distinct from other mutants with gastrulation failure, but is most similar to that of $\beta$-catenin, which affects both the WNT and NODAL pathways. A GSK3 $\beta$ inhibitor rescued the ability of mutant embryos to form extraembryonic mesoderm and partially rescued the mutant embryos' ability to form embryonic mesoderm. GSK3 can also affect adhesion complexes independent of WNT signaling. Given that Tenm4 is a cell adhesion molecule that acts at neuronal synapses in flies, it is possible that cell-cell interactions are perturbed in the mutants, preventing the communication required for gastrulation to proceed. Further work will be required to fully understand the signaling pathways involved in this intriguing and important molecule. 


\section{Methods}

Mouse strains and genotyping of adults and embryos $l 7 R n 3^{1777 S B}$ and $l 7 R n 3^{4323 S B}$ (renamed Tenm $4^{m 1}$ and $\mathrm{Tenm} 4^{m 4}$, respectively) originated at the Oak Ridge National Laboratory and were obtained from Dr. E. M. Rinchik. The mice are maintained as heterozygous stocks by backcrossing with the inbred strain FRCH/Rl. The mutant alleles were induced on the BALB/cRl background, and are tightly linked to the Tyr (albino) locus. Embryos from timed matings were examined to determine the characteristics of mutants. Noon of the day of the appearance of the vaginal plug was designated E0.5. Embryos were examined visually and photographed, or fixed for histology or in situ hybridization. DNA from each embryo was extracted for genotyping.

TOPGAL mice were purchased from The Jackson Laboratory. The mice are maintained as heterozygous stocks by backcrossing with the inbred strain FVB. To obtain doubly heterozygous mice $\left(\right.$ Tenm $4^{\mathrm{m} 1} /+$, topgal +), were crossed with Tenm $4^{m 1}$ heterozygous mice and tails from all albino F1 mice were collected for $\mathrm{x}$-gal staining. Furthermore, DNA from all x-gal positive tails (TOPGAL is expressed in the hair follicle) was extracted for genotyping.

Genotypes were determined by PCR analysis of genomic DNA from tail biopsies, embryonic yolk sac or whole embryos. Tails, yolk sacs or embryos were suspended in lysis buffer (50 mM Tris-HCl, pH 7.6, 20 mM NaCl, 0.1\% SDS and $0.2 \mathrm{mg} / \mathrm{ml}$ proteinase $\mathrm{K}$ ) and incubated at $55^{\circ} \mathrm{C}$ for $4-$ 16 hours and $95^{\circ} \mathrm{C}$ for 10 minutes. For genotyping of sectioned samples from histological analysis, portions of sections were scratched and incubated with $50 \mu \mathrm{l}$ of PCR reaction mix at $95^{\circ} \mathrm{C}$ for $10 \mathrm{~min}$, then analyzed by PCR. A $122 \mathrm{bp}$ fragment is amplified in BALB/cRl, $120 \mathrm{bp}$ in FVB and a $108 \mathrm{bp}$ in FRCH for D7Mit352 using the primers AGCCAATTGCAACCAAATTT (F) and AGCATGG AAAATTGACAATTCC (R). The PCR reaction was performed by denaturing at $94^{\circ} \mathrm{C}$ for 3 minutes, followed by 30 cycles of amplification: $94^{\circ} \mathrm{C} 1$ minute, $55^{\circ} \mathrm{C} 30$ seconds, $72^{\circ} \mathrm{C} 2$ minutes and final extension at $72^{\circ} \mathrm{C}$ for 5 minutes.

\section{Histology and whole mount in situ hybridization}

For histological examination, embryos were fixed in $4 \%$ paraformaldehyde overnight, embedded in paraffin wax, sectioned sagittally at 5-7 $\mu \mathrm{m}$ and stained with hematoxylin and eosin. For genotyping, parts of the sections were scratched prior to staining. Whole mount in situ hybridization using digoxigenin-labeled RNA probes was performed as described [61]. cDNA probes used for in situ hybridization were as described previously: Brachyury [23], Foxa2/HNF3 $\beta$ [26], Hesx1 [62], Lhx1 [27], Otx2 [28], and Bmp4 [29]. At least five mutant embryos were examined for each marker gene.

\section{BrdU labeling and TUNEL assays}

BrdU (100 $\mu \mathrm{g} / \mathrm{gram}$ of body weight) was injected intraperitoneally into pregnant females at E6.5. The females were sacrificed 20 min after injection and embryos were fixed with $4 \%$ paraformaldehyde, embedded in paraffin, and transversely sectioned at 5-7 $\mu \mathrm{m}$. The sections were stained with mouse anti-BrdU antibody (Sigma), visualized by reaction with $3,3^{\prime}$-diaminobenzidine and sections were counterstained with hematoxylin. For calculation of labeling index, we counted the BrdU positive cells in embryonic regions (5 slices from each embryo were used for average counting). TUNEL assays were performed with the In Situ Cell Death Detection Kit (Roche) and the sections were counterstained with methyl green.

\section{Embryonic teratoma assay}

E6.5 embryos were isolated from matings of heterozygotes. After removal of the ectoplacental cone, embryos were transplanted into the testes of sibling males. One or seven week(s) later, the testes were removed and were fixed in $4 \%$ paraformaldehyde, and embedded in paraffin for histological analysis as described above. For genotyping of embryo-derived teratomas, parts of the teratoma were scratched from sectioned paraffin blocks.

\section{Whole embryo cultures}

For whole embryo culture, embryos from matings between heterozygotes were dissected from decidual tissue, the parietal endoderm was removed and transferred to whole embryo culture medium containing (50\% DMEM + 50\% Rat serum obtained from Harlan) and placed into rolling culture. For the rescue experiment, E7.5 embryos from matings between heterozygotes were individually cultured as described above. 6-bromoindirubin- $3^{\prime}$-oxime (BIO) and 1methyl-6-bromoindirubin-3'-oxime (MeBIO) were purchased from Calbiochem/EMD Millipore (Billerica, MA). Some of cultured embryos were fixed with Carnoy's fixative for histology, and the others were fixed with $2 \%$ glutaraldehyde for X-gal staining. For the ectoplacental cone (EPC) culture, E7.5 embryos were dissected from decidual tissues and EPCs were isolated and cultured on Petri dish in the medium containing high-glucose DMEM $+10 \%$ fetal bovine serum for 3 hours to remove adult blood cells from the EPCs. The EPC explants were transferred to collagen-coated dishes with fresh medium and were further cultured for two days.

\section{Blastocyst outgrowth cultures}

Blastocysts (E3.5) were collected in M2 medium and then transferred to medium containing high-glucose DMEM, $20 \%$ fetal bovine serum, $0.1 \mathrm{mM} \beta$-mercaptoethanol, 1 $\mathrm{mM}$ sodium pyruvate, $1 \times$ non-essential amino acid, $2 \mathrm{mM}$ glutamine, 100 units of penicillin per $\mathrm{ml}$ and $0.1 \mathrm{mg}$ of streptomycin (ES medium lacking LIF) in 24-well plates 
coated with gelatin. The cultures were examined for attachment and growth, photographed and collected for genotyping.

\section{Immunohistochemistry}

Pou5f1, Snai1 and E-cadherin antibodies were purchased from Santa Cruz Biotechnology, Inc. (Santa Cruz, CA) and N-cadherin antibody (MNCD2) was provided from the Developmental Studies Hybridoma Bank (University of Iowa, IA). Paraffin-embedded sections were cleared in histoclear and rehydrated through an ethanol series to PBS. Endogenous peroxidase activity was quenched by incubation of sections with $6 \%$ hydrogen peroxide in methanol for 1 hour at room temperature. For immunostaining of embryo outgrowth cultures, cultures were fixed in methanol at $4^{\circ} \mathrm{C}$ for 10 minutes, treated with $6 \%$ hydrogen peroxide in methanol and rehydrated through a methanol series to PBS. The sections or culture cells were then blocked with 10\% normal sheep serum in PBS for an hour at room temperature and incubated with primary antibodies (diluted 1:500 - 1:2000) for an hour. After washing, the sections were incubated with horseradish-peroxidase-conjugated secondary antibodies diluted (1:200 - 1:1000) for an hour. The sections were then washed and reacted with peroxidase substrate.

For whole-mount immunostaining, embryos were fixed with $4 \%$ paraformaldehyde and transferred to methanol, treated with $6 \%$ hydrogen peroxide in methanol and rehydrated through a methanol series to PBS. Embryos were blocked with $10 \%$ normal sheep serum in PBS for 2 hours at room temperature and incubated with primary antibodies overnight at $4^{\circ} \mathrm{C}$. After five washes with PBS for an hour each, embryos were incubated with horseradish-peroxidase-conjugated secondary antibodies overnight at $4^{\circ} \mathrm{C}$, washed six times for an hour each and then placed in peroxidase substrate.

\section{Additional files}

Additional file 1: Figure S1. Variation of Brachyury expression in Tenm $4^{\mathrm{ml} / \mathrm{ml}}$ mutant embryos. No Brachyury expression was observed prior to E7.5 (see left panel). Some older Tenm $4^{\mathrm{ml} / \mathrm{ml}}$ mutant embryos (E8.5) show slight Brachyury expression (Right panel), which might be background staining. Alternatively, some transcription may occur in the extraembryonic portion of embryos homozygous for the Tenm $4^{m i}$ mutation. Tenm $4^{m 1 / m l}$ mutant embryo arrests at the gastrulation stage and fail to develop a mesoderm. Histological paraffin section prepared from Tenm $4^{\mathrm{ml} / \mathrm{ml}}$ mutant embryo, and stained with hematoxylin and eosin. Sagittal sections of Tenm $4^{\mathrm{mi} / \mathrm{ml}}$ embryo at day E7.5. Thickness of section is $7 \mathrm{~mm}$. Bar $100 \mu \mathrm{m}$.

Additional file 2: Figure S2. Blastocyst outgrowth culture. E3.5 blastocysts collected from heterozygote matings were cultured on gelatin-coated dishes, examined for proliferation and genotyped after culture. No differences between the genotypes were observed before culture. Poor trophoblast growth and impaired proliferation of ICM were observed in Tenm $4^{\mathrm{ml} / \mathrm{ml}}$ blastocyst culture. Photographs were taken at the first day of the culture $(A, B)$, the third day $(C, D)$ and the sixth day (E, F). A-C, wildtype blastocyst, D-F Tenm $4^{m 1 / m 1}$ mutant blastocyst.

\section{Competing interests}

The authors declare that they have no competing financial interests or noncompeting personal interests in the publication of the data in this manuscript. These data have not been reported elsewhere.

\section{Authors' contributions}

HN carried out the experimental manipulations and wrote the initial draft of the paper. MJJ provided mutants, funding, edited and wrote the paper. RNC edited and wrote the paper. All authors read and approved the final manuscript.

\section{Acknowledgments}

We thank Drs. Richard Behringer, Mary Dickinson, Christie Buchovecky and Amy C. Lossie for discussions prior to publication and helpful comments on the manuscript; Dr. Sharon Thomas for technical support in the early days of this project; Drs. Maki Wakamiya, Randy Johnson and Hiroki Kokubo for the in situ hybridization probes and for advice on in situ hybridization. The monoclonal antibody to $\mathrm{N}$-cadherin (developed by Dr. Takeichi) was obtained from the Developmental Studies Hybridoma Bank, the University of lowa. NIH grants U01 HD39372 and R01 CA115503 as well as a grant from the Kleberg Foundation to MJJ supported this work.

Received: 23 August 2012 Accepted: 19 March 2013

Published: 25 March 2013

\section{References}

1. Hogan B, Beddington R, Costantini F, Lacy E: In manipulating the mouse embryo. 2nd edition. Cold Spring Harbor, NY: Cold Spring Harbor Laboratory Press; 1994.

2. Nagy A, Gertsenstein M, Vintersten K, Behringer R: Manipulating the mouse embryo: a laboratory manual. Cold Spring Harbor, NY: Cold Spring Harbor Laboratory Press; 2002.

3. Tam PP, Behringer RR: Mouse gastrulation: the formation of a mammalian body plan. Mech Dev 1997, 68:3-25

4. Marikawa $Y$ : Wnt/beta-catenin signaling and body plan formation in mouse embryos. Semin Cell Dev Biol 2006, 17(2):175-184

5. Liu P, Wakamiya M, Shea MJ, Albrecht U, Behringer RR, Bradley A: Requirement for Wnt3 in vertebrate axis formation. Nat Genet 1999, 22:361-365.

6. Conlon FL, Lyons KM, Takaesu N, Barth KS, Kispert A, Herrmann B, Robertson EJ: A primary requirement for nodal in the formation and maintenance of the primitive streak in the mouse. Development 1994, 120:1919-1928.

7. Herrmann BG, Kispert A: The T genes in embryogenesis. Trends Genet 1994, 8:280-286.

8. Iannaccone PM, Zhou X, Khokha M, Boucher D, Kuehn MR: Insertional mutation of a gene involved in growth regulation of the mouse embryo. Dev Dynamics 1992, 194:198-208.

9. Baumgartner S, Martin D, Hagios C, Chiquet-Ehrismann R: Tenm, a Drosophila gene related to tenascin, is a new pair-rule gene. EMBO J 1994, 13:3728-3740.

10. Levine A, Bashan-Ahrend A, Budai-Hadrian O, Gartenberg D, Menasherow S, Wides R: Odd Oz: a novel Drosophila pair rule gene. Cell 1994, 77:587-598.

11. Tucker RP, Chiquet-Ehrismann R: Teneurins: a conserved family of transmembrane proteins involved in intercellular signaling during development. Dev Biol 2006, 290(2):237-245.

12. Zheng L, Michelson Y, Freger V, Avraham Z, Venken KJ, Bellen HJ, Justice MJ, Wides R: Drosophila Ten-m and filamin affect motor neuron growth cone guidance. PLoS One 2011, 6(8):e22956.

13. Mosca TJ, Hong W, Dani VS, Favaloro V, Luo L: Trans-synaptic Teneurin signalling in neuromuscular synapse organization and target choice. Nature 2012, 484(7393):237-241.

14. Oohashi T, Zhou XH, Feng K, Richter B, Morgelin M, Perez MT, Su WD, Chiquet-Ehrismann R, Rauch U, Fassler R: Mouse ten-m/Odz is a new family of dimeric type II transmembrane proteins expressed in many tissues. J Cell Biol 1999, 145:563-577.

15. Wang XZ, Kuroda M, Sok J, Batchvarova N, Kimmel R, Chung P, Zinszner H, Ron $D$ : Identification of novel stress-induced genes downstream of chop. EMBO J 1998, 17:3619-3630.

16. Lossie AC, Nakamura H, Thomas SE, Justice MJ: Mutation of I7Rn3 shows that Odz4 is required for mouse gastrulation. Genetics 2005, 169:285-299.

17. Zhou XH, Brandau O, Feng K, Oohashi T, Ninomiya Y, Rauch U, Fassler R: The murine Ten-m/Odz genes show distinct but overlapping expression patterns during development and in adult brain. Gene Expr Patterns 2003, 3:397-405. 
18. Nolan PM, Kapfhamer D, Bucan M: Random mutagenesis screen for dominant behavioral mutations in mice. Methods 1997, 13(4):379-395.

19. Rinchik EM, Carpenter DA, Selby PB: A strategy for fine-structure functional analysis of a 6- to 11-centimorgan region of mouse chromosome 7 by high-efficiency mutagenesis. Proc Natl Acad Sci USA 1990, 87:898-900.

20. Rinchik EM: Chemical mutagenesis and fine-structure functional analysis of the mouse genome. Trends Genet 1991, 7:15-21.

21. Rinchik EM, Carpenter DA: N-ethyl-N-nitrosourea mutagenesis of a 6- to 11-cM subregion of the Fah-Hbb interval of mouse chromosome 7: Completed testing of 4557 gametes and deletion mapping and complementation analysis of 31 mutations. Genetics 1999, 152:373-383.

22. Herrmann BG: Expression pattern of the Brachyury gene in whole-mount TWis /TWis mutant embryos. Development 1991, 113:913-917.

23. Kispert A, Hermann BG: The Brachyury gene encodes a novel DNA binding protein. EMBO J 1993, 12:3211-3220.

24. Blum M, Gaunt SJ, Cho KWY, Steinbeisser H, Blumberg B, Bittner D, De Robertis EM: Gastrulation in the mouse: the homeobox gene goosecoid. Cell 1992, 69:1097-1106.

25. Ang SL, Wierda A, Wong D, Stevens KA, Cascio S, Rossant J, Zaret KS: The formation and maintenance of the definitive endoderm lineage in the mouse: involvement of HNF3/forkhead proteins. Development 1993, 119:1301-1315

26. Sasaki H, Hogan BL: Differential expression of multiple fork head related genes during gastrulation and axial pattern formation in the mouse embryo. Development 1993, 118:47-59.

27. Barnes JD, Crosby JL, Jones CM, Wright CVE, Hogan BL: Embryonic expression of Lim-1, the mouse homolog of Xenopus Xlim-1, suggests a role in lateral mesoderm differentiation and neurogenesis. Dev Biol 1994, 161:168-178.

28. Ang SL, Jin O, Rhinn M, Daigle N, Stevenson L, Rossant J: A targeted mouse Otx2 mutation leads to severe defects in gastrulation and formation of axial mesoderm and to deletion of rostral brain. Development 1996, 122:243-252.

29. Winnier G, Blessing M, Labosky PA, Hogan BL: Bone morphogenetic protein-4 is required for mesoderm formation and patterning in the mouse. Genes Dev 1995, 9:2105-2116.

30. Solter D, Skreb N, Damjanov I: Extrauterine growth of mouse egg cylinders results in malignant teratoma. Nature 1970, 227:503-504

31. Diwan SB, Stevens LC: Development of teratomas from the ectoderm of mouse egg cylinders. J Natl Cancer Inst 1976, 57:937-942.

32. Rosner MH, Vigano MA, Ozato K, Timmons PM, Poirier F, Rigby PW, Staudt LM: A POU-domain transcription factor in early stem cells and germ cells of the mammalian embryo. Nature 1990, 345:686-692.

33. Okamoto K, Okazawa H, Okuda A, Sakai M, Muramatsu M, Hamada H: A novel octamer binding transcription factor is differentially expressed in mouse embryonic cells. Cell 1990, 60:461-472.

34. Pesce $M$, Wang $X$, Wolgemuth DJ, Scholer H: Differential expression of the Oct-4 transcription factor during mouse germ cell differentiation. Mech Dev 1998, 71:89-98.

35. Scholer HR, Ruppert S, Suzuki N, Chowdhury K, Gruss P: New type of POU domain in germ line-specific protein Oct-4. Nature 1990, 344:435-439.

36. Yeom Yl, Fuhrmann G, Ovitt CE, Brehm A, Ohbo K, Gross M, Hubner K Scholer HR: Germline regulatory element of Oct-4 specific for the totipotent cycle of embryonal cells. Development 1996, 122:881-894.

37. DasGupta R, Fuchs E: Multiple roles for activated LEF/TCF transcription complexes during hair follicle development and differentiation. Development 1999, 126:4557-4568.

38. Valvezan AJ, Klein PS: GSK-3 and Wnt Signaling in Neurogenesis and Bipolar Disorder. Front Mol Neurosci 2012, 5:1.

39. Sato N, Meijer L, Skaltsounis L, Greengard P, Brivanlou AH: Maintenance of pluripotency in human and mouse embryonic stem cells through activation of Wnt signaling by a pharmacological GSK-3-specific inhibitor. Nat Med 2004, 10:55-63.

40. Gu Z, Nomura M, Simpson BB, Lei H, Feijen A, van den Eijnden-van Raaji J, Donahoe PK, Li E: The type I activin receptor ActRIB is required for egg cylinder organization and gastrulation in the mouse. Genes Dev 1998, 12:844-857.

41. Sirard C, de la Pompa JL, Elia A, Itie A, Mirtsos C, Cheung A, Hahn S, Wakeham A, Schwartz L, Kern SE, Rossant J, Mak TW: The tumor suppressor gene Smad4/Dpc4 is required for gastrulation and later for anterior development of the mouse embryo. Genes Dev 1998, 12:107-119.
42. Waldrip WR, Bikoff EK, Hoodless PA, Wrana JL, Robertson EJ: Smad2 signaling in extraembryonic tissues determines anterior-posterior polarity of the early mouse embryo. Cell 1998, 92:797-808

43. Weinstein M, Yang X, Li C, Xu X, Gotay J, Deng CX: Failure of egg cylinder elongation and mesoderm induction in mouse embryos lacking the tumor suppressor smad2. Proc Natl Acad Sci USA 1998, 95:9378-9383.

44. James D, Levine AJ, Besser D, Hemmati-Brivanlou A: TGFbeta/activin/nodal signaling is necessary for the maintenance of pluripotency in human embryonic stem cells. Development 2005, 132:1273-1282.

45. Chen X, Weisberg E, Fridmacher V, Watanabe M, Naco G, Whitman M: Smad4 and FAST-1 in the assembly of activin-response factor. Nature 1997, 389:85-89.

46. Watanabe M, Whitman M: FAST-1 is a key maternal effector of mesoderm inducers in the early Xenopus embryo. Development 1999, 126:5621-5634.

47. Mishina Y, Suzuki A, Ueno N, Behringer RR: Bmpr encodes a type I bone morphogenetic protein receptor that is essential for gastrulation during mouse embryogenesis. Genes Dev 1994, 9:3027-3037.

48. Coucouvanis E, Martin GR: BMP signaling plays a role in visceral endoderm differentiation and cavitation in the early mouse embryo. Development 1999, 126:535-546.

49. Hsieh JC, Lee L, Zhang L, Wefer S, Brown K, DeRossi C, Wines ME, Rosenquist T, Holdener BC: Mesd encodes an LRP5/6 chaperone essential for specification of mouse embryonic polarity. Cell 2003, 112:355-367.

50. Kelly OG, Pinson Kl, Skarnes WC: The Wnt co-receptors Lrp5 and Lrp6 are essential for gastrulation in mice. Development 2004, 131:2803-2815.

51. Zeng L, Fagotto F, Zhang T, Hsu W, Vasicek TJ, Perry WL, Lee JJ, Tilghman SM, Gumbiner BM, Constantini F: The mouse Fused locus encodes Axin, an inhibitor of the Wnt signaling pathway that regulates embryonic axis formation. Cell 1997, 90:181-192.

52. Behrens J, Jerchow BA, Würtele M, Grimm J, Asbrand C, Wirtz R, Kühl M, Wedlich D, Birchmeier W: Functional interaction of an Axin Homolog Conductin, with b-Catenin, APC, and GSK3 b. Science 1998, 280:596-599.

53. Moekel M, Huelsken J, Wakamiya M, Ding J, van de Wetering M, Clevers $H$ Taketo MM, Behringer RR, Shen MM, Birchmeier W: b-catenin regulates Cripto- and Wnt3-dependent gene expression programs in mouse axis and mesoderm formation. Development 2003, 130:6283-6294.

54. Huelsken J, Vogel R, Brinkmann V, Erdmann B, Birchmeier C, Birchmeier W: Requirement for beta-catenin in anterior-posterior axis formation in mice. J Cell Biol 2000, 148:567-578.

55. Wray J, Hartmann C: WNTing embryonic stem cells. Trends Cell Biol 2012, 22(3):159-168

56. Ciruna B, Rossant J: FGF signaling regulates mesoderm cell fate specification and morphogenetic movement at the primitive streak. Dev Cell 2001, 1:37-49.

57. Grimes CA, Jope RS: The multifaceted roles of glycogen synthase kinase 3beta in cellular signaling. Prog Neurobiol 2001, 65(4):391-426.

58. Clark CE, Nourse CC, Cooper HM: The tangled web of non-canonical Wnt signalling in neural migration. Neurosignals 2012, 20(3):202-220.

59. Okerlund ND, Cheyette BN: Synaptic Wnt signaling-a contributor to major psychiatric disorders? J Neurodev Disord 2011, 3(2):162-174

60. Sklar P, Ripke S, Scott LJ, Andreassen OA, Cichon S, Craddock N, Edenberg HJ, Nurnberger JI Jr, Rietschel M, Blackwood D, et al: Large-scale genomewide association analysis of bipolar disorder identifies a new susceptibility locus near ODZ4. Nat Genet 2011, 43(10):977-983.

61. Echelard Y, Epstein DJ, St-Jacques B, Shen L, Mohler J, McMahon JA McMahon AP: Sonic hedgehog, a member of a family of putative signaling molecules, is implicated in the regulation of CNS polarity. Cell 1993, 75:1417-1430.

62. Thomas $\mathrm{P}$, Beddington R: Anterior primitive endoderm may be responsible for patterning the anterior neural plate in the mouse embryo. Current biology: CB 1996, 6(11):1487-1496.

doi:10.1186/1471-213X-13-9

Cite this article as: Nakamura et al.: Mouse Tenm4 is required for mesoderm induction. BMC Developmental Biology 2013 13:9. 